\title{
EFECTO DEL FLUFENOXURON SOBRE LA ACTIVIDAD COPULADORA DEL MACHO DE SPODOPTERA LITTORALIS (BOISD.) (LEPIDOPTERA: NOCTUIDAE) ${ }^{1}$
}

\author{
JAIRO ROBERTO MENDONÇA LYRA² ${ }^{2}$ JOSÉ MARIA GUZMAN FERRAZZ ${ }^{3}$ y ANA PAULA PEREIRA DA SILVA ${ }^{4}$
}

RESUMEN - Ha sido evaluado el efecto de benzoilfenylureia sobre la capacidad copuladora de machos de Spodoptera littoralis (Boisd.) (Lepdoptera: Noctuidae). Dos grupos de larvas de $3^{\circ}$ estadio fueron tratados con el flufenoxuron, uno por ingestión y el otro por contacto, con una concentración del producto igual a la DL60. Cuando hubo la emergencia de los adultos, 10 machos por tratamiento fueron individualizados en cilindros de papel de filtro y puestos a copular con hembras vírgenes, que eron sustituídas diariamente, hasta la muerte de las mismas. Las hembras que fueron ofrecidas a los machos, al morir, eron dissecadas para verificar se estaban o no copuladas, a través de la observación de la presencia de espermatóforos en la bursa copulatrix. Fue observado que los machos de S. littoralis provenientes de larvas tratadas con el flufenoxuron, no tuvieron su capacidad copuladora alterada. La metodología fue considerada adecuada para evaluar la atividad copuladora y el número de copulaciones realizadas por el macho de esta especie.

Términos para índice: lucha química, gusano del algodón, fertilidad.

\section{FLUFENOXURON EFFECTS ON COPULATING CAPACITY OF SPODOPTERA LITTORALIS (BOISD.) (LEPIDOPTERA: NOCTUIDAE) MALES}

\begin{abstract}
The effect of benzoilphenylureas was studied on the copulating capacity of Spodoptera littoralis (Boisd.) (Lepidoptera: Noctuidae) males. Two groups of third instar larvae were treated with flufenoxuron, one by ingestion and another by contact with an DL60 rate. After emergence of the adults, ten males of the each treatment were put individualy in a filter paper cylinder, from the first night until death, in the presence of a virgin female that shift daily. After the female death they were desiccated in order to see the presence or not of the spermatophore in the bursa copulatrix. It was observed that the $S$. littoralis males that proceeded from the flufenoxuron treated larvae did not change their copulating capacity. The metodology was considerate efficient to evaluate the copulate capacity of this specie males.
\end{abstract}

Index terms: chemical control, cotton leafworm, fertility.

\footnotetext{
${ }^{1}$ Aceptado para publicación en 30 de julio de 1998.

${ }^{2}$ Ing. Agr., Ph.D., Dep. de Agronomia, Universidade Federal de Uberlândia, Campus Umuarama, Av. Amazonas, s/noำ, bloco 2E, sala 13, CEP 38400-902 Uberlândia, MG. E-mail: jairo@ufu.br

${ }^{3}$ Biólogo, Ph.D., Embrapa-Centro Nacional de Pesquisa de Monitoramento e Avaliação de Impacto Ambiental (CNPMA), Rod. SP 340, km 127,5, Caixa Postal 69, C E P $13820-000 \mathrm{~J}$ a u ariúna, S P. E-mai 1: ferraz@cnpma.embrapa.br

${ }^{4}$ Ing. Agr., Escola Agrotécnica Federal de Uberlândia, Fazenda Sobradinho, Zona rural, CEP 38409-970 Uberlândia, MG.
}

\section{INTRODUCCIÓN}

El descubrimiento de la acción insecticida de los inhibidores del desarrollo de los insectos (IDI) del grupo de las benzoilfenilureas aporta una nueva alternativa en la lucha contra las plagas agrícolas. El efecto larvicida de estos compuestos ha sido comprobado en diversas especies de insectos (Mulder \& Gijswijh, 1973; Guyer \& Neumann, 1988; Omatsu et al., 1991; Degheele et al., 1993) e de igual modo, el efecto a más largo plazo en los individuos que sobreviven al tratamiento (Sehnal et al., 1986; Prasad et al., 1992). 
La reducción del potencial reprodutor en especies de insectos de interés agrícola y que sobreviven al tratamiento con insecticidas se considera un efecto de gran importancia en el marco de un sistema de lucha integrada. En Spodoptera littoralis (Boisd.) (Lepidoptera: Noctuidae), la aplicación de VPN (Baculoviridae) no parece afectar la actividad copuladora ni la producción de espermatóforos de machos tratados, aunque sí al porcentaje de viabilidad de huevos (Santiago Álvarez \& Vargas Osuna, 1987). En la misma especie la fecundidad de hembras y la eclosión de huevos se reducen significativamente en larvas que sobreviven al tratamiento con flufenoxuron (Aldebis et al., 1988). Los mismos autores estudiando el efecto del fenoxicarb y metopreno en el desarrollo del sistema reproductor interno y de células germinativas en larvas de 50 estadio de esa misma especie observaron un retraso en la fusión testicular; además en prepupas procedentes de larvas tratadas con fenoxicarb aparecen alteraciones en el desarrollo de las células germinales que conducen a malformaciones de espermatozoides y paquetes de esperma eupirenos (Aldebis et al., 1994).

Dado la importancia de la especie plaga $S$. littoralis y del efecto de esterilidad que puede presentar la aplicación de los inhibidores de la síntesis de la quitina, nos ha parecido de gran interés evaluar el efecto de la aplicación del flufenoxuron (materia activa) sobre la capacidad copuladora y la producción de espermatóforos por el macho de esta especie.

\section{MATERIAL Y MÉTODOS}

Han sido empleadas larvas de S. littoralis de 3 o estadio procedentes de poblacción mantenida en insectario $\left(26^{\circ} \mathrm{C} \pm 2 ; 65 \pm 5 \%\right.$ HR y 16 horas/luz) sobre medio artificial (Santiago Álvarez, 1977). Las mismas procedían de larvas recogidas en cultivos de alfafa en octubre de 1991 en la provincia de Córdoba (España). Todos los ensayos se llevaron a acabo en la Unidad de Entomología de la Universidad de Córdoba (España) en 1995, bajo las mismas condiciones de temperatura y humedad.
El producto utilizado fue el flufenoxurón producto técnico $(99,5 \%$ m.a.) y como diluyente la acetona. En el tratamiento de las larvas, tanto por ingestión como por contacto, se siguió el método descrito por Lyra (1994). Se utilizarón 240 larvas de 3e estadio con una concentración equivalente a la $\mathrm{DL}_{60}$ del producto $\left(10^{-3} \mu \mathrm{g} \mathrm{g} /\right.$ larva y $2,94 \times 10^{-4} \mu \mathrm{g}$ g/larva por ingestión y contacto respectivamente) y se utilizaron 80 larvas como testigo. El objetivo de se utilizar una gran cantidad de larvas fue solamente para se tener un número considerable de adultos para formar las combinaciones. El tratamiento larvario consistió en tratamientos por ingestión y contacto con el producto. Para el tratamiento por ingestión, se depositó, por medio de un microaplicador, una gota de $2 \mathrm{~mL}$ de la solución sobre redondeles de hoja de alfafa de $5 \mathrm{~mm}$ de diámetro que se ofreceron a larvas individualizadas en cajas de plástico transparente, de $26 \mathrm{~mm}$ de diámetro y $13 \mathrm{~mm}$ de altura, con tapa cerrada. A las larvas testigo se les ofreceron redondeles tratados con una gota de acetona de igual volumen. Respecto a la aplicación tópica, también por medio de un microaplicador se depositó una gota de $2 \mathrm{~mL}$ de volumen en la parte dorsal de la región torácica de cada una de las larvas, individualizándolas en cajas de plástico transparente de $26 \mathrm{~mm}$ de diámetro y $13 \mathrm{~mm}$ de altura, con tapa cerrada. Cada caja contuvo un redondel de hoja de alfafa de $5 \mathrm{~mm}$ de diámetro. A las larvas testigo se les trató de igual forma con acetona. En ambos tratamientos las larvas que en el plazo de 24 horas no habían consumido el redondel tratado fueron eliminadas, las restantes se transfiereron a nuevas cajas con tapa cerrada que permitió la aireación, y fueron alimentadas con dieta artificial.

En los dos tipos de tratamiento, cada 24 horas se realizaron controles para anotar el número de larvas muertas y el estadio en que tuvo lugar la muerte.

Cuando hubo mortalidad en las larvas testigo, los porcentajes de mortalidad de cada dosis fueron corregidos mediante la fórmula de Abbott (1925). Al mismo tiempo un grupo de 1000 larvas se mantuvieron en las mismas condiciones que las larvas tratadas con el objeto de obtener el suficiente número de hembras para los apareamientos. Cuando los adultos emergían, los machos se confinaron individualizados en cilindros de papel de filtro de $12 \mathrm{~cm}$ de diámetro y $24 \mathrm{~cm}$ de altura, provistos y su fondo de un recipiente con agua mielada como alimento.

De este modo diez machos de cada un de las condiciones de tratamientos y idéntico número de los correspondientes testigos se aparearon a partir de la primera noche de su emergencia, con una hembra virgen de dos días de edad que diariamente se cambiaba hasta la muerte de cada macho; 
todas las hembras se diseccionaron para observar la presencia o no de espermatóforos en la bursa copulatrix. La disección se llevó a cabo separando con un bisturí el abdomen de la hembra e introduciéndolo en solución de Belar cuya composición fue la siguiente: $\mathrm{NaCl}=6,00 \mathrm{~g}$, $\mathrm{CaCl}_{2}=0,29 \mathrm{~g}, \mathrm{Na}_{2} \mathrm{CO}_{3}=0,29 \mathrm{~g}$, agua destilada $1 \mathrm{~L}$.

Con unas pinzas de disección se extrayó cuidadosamente la genitalia interna de la hembra, en que se observó en microscópico la presencia o no de espermatóforos en la bursa copulatrix.

El estudio estadístico de los datos se llevó a cabo a través del análisis de varianza y comparación de médias por el test de Tukey al nivel de $1 \%$ de probabilidad.

\section{RESULTADOS Y DISCUSIÓN}

En el Cuadro 1 se encuentra la média de espermatóforos producidos por cada macho quando se le ofrecía diariamente una hembra virgen de dos días de edad.

Los machos no tratados copularon en el transcurso de su vida con un número de hembras que osciló entre una média de 4,8 hembras, valor ligeramente inferior al de los tratados por contacto o ingestión, cuya média de copulaciones fue de 5,6 y 5,2 respectivamente, aunque estas diferencias no alcanzaron significación estadística.

Se observó que algunos individuos, el 30\% de los tratados por contacto y el $10 \%$ de los tratados por ingestión, fueron capaces de copular dos veces en un mismo día. Estas dobles copulaciones representaron, respecto del número total de hembras copuladas, un 5,36\% en el grupo de los machos tratados por contacto y un $1,92 \%$ en el de machos tratados por ingestión. Se observó copulación hasta el 9o dia en los machos tratados por ingestión o contacto $\mathrm{y}$ en los machos tratados los testigos solo hasta el $6^{\circ}$ dia.

Los machos que proceden de larvas tratadas, por ingestión o contacto con flufenoxuron, copulan reiteradas veces a lo largo de su vida, mostrando un comportamiento similar al de los normales de esta especie (Kehat \& Gordon, 1975) y de la especie próxima S. litura (Miyashita \& Fuwa, 1972). Realizan las copulaciones en días sucesivos o en días alternos, aunque para todos los grupos es mayor la tendencia a copular en días sucesivos. La copulación a intervalos parece estar influida por condiciones fisiológicas de la hembra con quien se aparean en cada momento (Fujjie \& Miyashita, 1973).

La producción total de espermatóforos a lo largo de la vida de cada uno de los machos, procedentes de larvas tratadas, está sometida a variabilidad individual al igual que en los machos testigos (Kehat \& Gordon, 1975). El número medio de espermatóforos producidos por los machos procedentes de larvas tratadas y el de los testigos, que no difieren significativamente, se corresponden con los obtenidos para esta misma especie por otros autores (Kehat \& Gordon, 1975; Vargas Osuna \& Santiago Álvarez, 1985); en consecuencia, los machos de $S$. littoralis que proceden de larvas tratadas con flufenoxuron no tienen alterada su capacidad copuladora, pudiendo competir sexualmente con los normales.

CUADRO 1. Número médio de espermatóforos a lo largo de la vida de los machos de Spodoptera littoralis procedentes de larvas $L_{3}$ que habían sido tratadas con el flufenoxuron por ingestión y contacto $\left(26^{\circ} \mathrm{C} \pm 2 ; 65 \pm 5 \%\right.$ HR y 16 horas/luz).

\begin{tabular}{lccccc}
\hline Tratamiento & $\begin{array}{c}\mathrm{N}^{\mathrm{o}} \text { de } \\
\text { machos }\end{array}$ & $\begin{array}{c}\mathrm{N}^{\underline{\mathrm{o}}} \text { total de hembras } \\
\text { puestas a copular }\end{array}$ & $\begin{array}{c}\mathrm{N}^{\mathrm{o}} \text { total de hembras } \\
\text { copuladas }\end{array}$ & $\begin{array}{c}\mathrm{N}^{\mathrm{o}} \text { de machos que copulan } \\
\text { más de una vez }\end{array}$ & $\begin{array}{c}\mathrm{N}^{\mathrm{o}} \text { médio de espermatóforos } \\
\text { encontrados (média de copulaciones) }\end{array}$ \\
\hline Testigo & 10 & 85 & 48 & 0 & $4,8 \mathrm{a}$ \\
Ingestión & 10 & 95 & 52 & 1 & $5,2 \mathrm{a}$ \\
Contacto & 10 & 93 & 56 & 3 & $5,6 \mathrm{a}$ \\
\hline
\end{tabular}

${ }^{1}$ Médias seguidas de la misma letra no differen entre sí por el test de Tukey al nível de 1\% de probabilidad. 


\section{CONCLUSIONES}

1. Los machos de Spodoptera littoralis que proceden de larvas tratadas por ingestión o contacto con flufenoxuron no tienen alterada su capacidad copuladora.

2. La metodología de disección de las hembras y observación de presencia o no de espermatóforos en la bursa copulatrix se mostró adequada para avaliar la actividad copuladora del macho de $S$. littoralis, podendo la misma ser utilizada en estudios de fertilidad en otros noctuideos.

\section{AGRADECIMIENTOS}

Al CNPq, por la beca de investigación, y a la Universidad de Córdoba (España) por suministrar los medios necesarios para realizar dicho trabajo; a la periodista Maria Cristina Tordin y a la bibliotecaria Maria Amélia de Toledo Leme por la ayuda en las tarefas de revisión y escrita.

\section{REFERENCIAS}

ABBOTT, W.S. A method of computing the effectiveness of an insecticide. Journal of Economic Entomology, v.18, p.265-267, 1925.

ALDEBIS, H.K.; VARGAS OSUNA, E. ; SANTIAGO ÁLVAREZ, C. Efectos del fenoxicarb y del metopreno sobre el desarrollo del sistema reproductor interno del macho de Spodoptera littoralis (Boisduval) (Lepidoptera: Noctuidae). Investigación Agropecuária: Protección Vegetal, v.9, n.2, p.281-287, 1994.

ALDEBIS, H.K.; VARGAS OSUNA, E.; SANTIAGO ÁLVAREZ, C. Respuesta de Spodoptera littoralis (Boisd.) (Lepidoptera: Noctuidae) al flufenoxurón, un regulador del crecimiento de los insectos, aplicado a larvas de quinto estadio. Boletin de Sanidad Vegetal Plagas, v.14, p.157-161, 1988.

DEGHEELE, D.; YI, S.X.; BAI, C. Oxicity of benzoylphenylureas to the african armyworm Spodoptera exempta (Walker). Crop Protection, v.12, n.2, p.35-38, 1993.

FUJJIE, A.; MIYASHITA, K. Further studies on the reiterative mating ability in males of Spodoptera litura, F. (Lepidoptera: Noctuidae). Applied Entomology and Zoology, v.8, p.131-137, 1973.
GUYER. W.; NEUMANN, R. Activity and fate of chlorfluazuron and diflubenzuron in the larvae of Spodoptera littoralis and Heliothis virescens. Pesticide Biochemistry and Physiology, v.30, p.166-177, 1988.

KEHAT, M.; GORDON, D. Mating, longevity, fertility and fecundity of cotton leafworm, Spodoptera littoralis (Boisd.). Phytoparasitica, v.3, p.87-102, 1975.

LYRA, J.R.M. Acción por ingestión y contacto del flufenoxuron sobre larvas de Agrotis segetum (Den. et Schiff.) y Spodoptera littoralis (Boisd.) (Lepidoptera: Noctuidae). Boletin de Sanidad Vegetal Plagas, v.20, p.45-48, 1994.

MIYASHITA, K.; FUWA, M. The occurrence time, reiterative ability and duration of mating Spodoptera litura F. (Lepidoptera: Noctuidae). Applied Entomology and Zoology, v.7, p.171-173, 1972.

MULDER, R.; GIJSWIJT, M. J. The laboratory evaluation of two promising new insecticides wich interfere with cuticle deposition. Pesticide Science, v.4, n.5, p.737-745, 1973.

OMATSU, M.; YOSHIDA, K.; TOKI, T. Development of malformed larvae induced by a benzoylphenyl urea insecticide, chlorfluazuran in the commom cutworm Spodoptera litura (Fabricius). Journal of Pesticide Science, v.16, p.189-194, 1991.

PRASAD, S.; SRIVASTAVA, B.B.L.; SINGH, R.B.; SINGH, D.R. Effect of diflubenzuron on biotic potential of Spodoptera litura (Fabr.) Biodiversity, v.3, n.1, p.87-90, 1992.

SANTIAGO ÁlVAREZ, C.; VARGAS OSUNA, E. Acción de la infección por el vírus de la poliedrosis nuclear (Baculoviridae) sobre la capacidad copuladora del macho de Spodoptera littoralis (Boisd.) (Lep. Noctuidae). Boletin de Sanidad Vegetal Plagas, v.13, p.167-171, 1987.

SANTIAGO ÁlVAREZ, C. Vírus de insectos: multiplicación, aislamiento y bioensayo de baculovírus. [S.1.]: Fundación Juan March, 1977.99p. (Serie Universitária, 43).

SEHNAL, F.; KANDIL, M.A.; SAMMOUR, E.L.; HAM, A. Sterilization of Spodoptera littoralis (Lepidoptera) with diflubenzuron and trifluron administered to larvae. Acta Entomologica Bohemoslovaca, v.83, p.253-259, 1986.

VARGAS OSUNA, E.; SANTIAGO ÁlVAREZ, C. Evolución de los ovarios de hembras adultas de S. littoralis (Boisd.) (Lepidoptera: Noctuidae). Boletim da Sociedade Portuguesa de Entomologia, v.3, p.145-152, 1985. Suplemento 1. 